\title{
Gene environment interaction of GALNT2 and APOE gene with hypertension in the Chinese Han Population ${ }^{1}$
}

\author{
Xuejuan Zhang ${ }^{\mathrm{a}}$, Haiping Zhao ${ }^{\mathrm{b}}$, Jietao Zhang ${ }^{\mathrm{a}}$, Di Han ${ }^{\mathrm{a}}$, Yu Zheng ${ }^{\mathrm{a}}$, Xiaozi Guo ${ }^{\mathrm{a}}$, Dian He ${ }^{\mathrm{b}}$, \\ Jin Guo ${ }^{\mathrm{b}, *}$ and Yingcui Wang ${ }^{\mathrm{c}, *}$ \\ ${ }^{a}$ General Medicine, Affiliated Hospital of Qingdao University, China \\ ${ }^{b}$ School of Public Health, Capital Medical University, 10 Xitoutiao, Youanmen Wai, Fengtai Dist, \\ Beijing, China \\ ${ }^{c}$ Cardiovascular Centre, Qilu Hospital, Shandong University, No.758, Hefei Road, Shibei Dist, \\ Qingdao, Shandong, China
}

\begin{abstract}
In some GWAs studies, GALNT2 and APOE polymorphisms have been identified to be related to alterations of plasma or serum HDL-C and TG concentrations. The purpose of our study is to assess the contribution of GALNT2 rs4846914, APOE rs429358, rs7412, rs405509 variants, and several environmental factors to the development of hypertension disease in the China Han population. A hospital-based case-control study was conducted. Cases were hypertension $(n=211)$ and controls were normal participants $(n=434)$. The AA, AG, and GG genotype frequencies of GALNT2 rs 4846914 were $22.8 \%, 43.1 \%$, and $34.1 \%$ in hypertension subjects, and $35.3 \%, 44.2 \%$, and $20.5 \%$ in controls $(P<0.05)$, respectively. The OR of the AG genotype adjusted for all risk factors compared to the AA genotype was 1.61 (95\%CI: 1.02 to 2.56 ) and to the GG genotype 2.67 (95\%CI: 1.59 to 4.488 ). There was no significant difference between the APOE rs429358, rs7412, and rs405509 genotype frequencies in hypertension and control subjects. The present work indicates that SNP rs4846914 in GALNT2 gene is related to an increased risk of hypertension in China Han population, but the APOE gene is not.
\end{abstract}

Keywords: Gene polymorphisms, GALNT2, APOE, hypertension, China

\section{Introduction}

Hypertension is one of serious risk elements and the main causes of cardiovascular disease (CVD) worldwide, and it is a rising threat to Chinese public health. Reports indicated that the quantities of hypertension patients in China have grown from less than 100 million in 1991 to 260 million in 2012 [1]. Three previous surveys conducted in 1959, 1979 and 1991 and a report on CVD (2007) show that

\footnotetext{
${ }^{1}$ The first two authors contributed equally to this study. Moreover, there is no competing interest in this paper.

*Address for correspondence: Jin Guo, School of Public Health, Capital Medical University, 10 Xitoutiao, Youanmen Wai, Fengtai Dist, Beijing, China. Tel.: +86-010-83911778; Fax: +86-010-83911778; E-mail: guojin5827501@163.com.

Yingcui Wang, Cardiovascular Centre, Qilu Hospital, Shandong University, No.758, Hefei Road, Shibei Dist, Qingdao, Shandong, China. Tel.: +86-0532-66850662; Fax: +86-0532-81726339; Email: 13963975615@163.com.
} 
the prevalence of hypertension grew dramatically from $5.11 \%$ in 1959 to $17.65 \%$ in 2002 [2]. More epidemiologic evidence exists to support the fact that the prevalence of hypertension is increasing [3].

The single nucleotide polymorphisms (SNP) rs4846914 is an intronic variant of the UDP-N-acetyl-alpha-D-galactosamine polypeptide N-acetylgalactosaminyltransferase 2 (GALNT2) gene. The minor G allele of SNP rs4846914 is related to higher triglyceride enrichment [4]. GALNT2 gene is mapped locus in $150 \mathrm{~kb}$ of the lead SNP on chromosome 1q42 region, and the lead SNP lies in an intron of the gene [5]. Some previous GWAs studies have indicated that GALNT2 polymorphisms are related to alterations of plasma or serum HDL-C [6-8] and TG $[9,10]$ concentrations $[11,12]$.

Apolipoprotein E (ApoE), along with ApoA, ApoB, ApoC, ApoD, ApoM, ApoH, ApoJ, and ApoL are members of the apolipoprotein gene family [13]. The APOE gene is composed of 4 exons and 3 introns, spans 3597 nucleotides, and encodes a 299-amino acid polypeptide. This gene lies on chromosome 19q13.2 region and is tightly connected to the ApoC-I/C-II gene complex [14]. ApoE is mainly produced in the liver, but other tissues or organs such as the brain, kidneys, adrenals, spleen, macrophages, and gonads also produce this protein [15]. Accumulating evidence reveals that the APOE gene polymorphism is genetically related to many diseases.

The main purpose of our research is to explore the contribution of GALNT2 rs4846914, APOE rs429358, rs7412, rs405509 variants, and several environmental factors to the development of hypertension disease in China Han population.

\section{Materials and methods}

\subsection{Study populations}

This research was performed in China and coordinated by the affiliated hospital of Capital Medical University, Beijing. It was authorized by the Ethics Committee of Capital Medical University, and informed consent acquired from the controls and patients. A patient was diagnosed with hypertension if they had a blood pressure $\geq 140 / 90 \mathrm{~mm} \mathrm{Hg}$, if they were on antihypertensive therapy, or if they had a documented history of hypertension. At least two age- $( \pm 5$ years) control (without a history of hypertension) was matched for each case.

\subsection{Epidemiological survey}

A standardized manner was applied to manage the organized questionnaires and physical examinations by trained personnel. Normative questionnaires were applied to gather the information about demographics, socialeconomic status, lifestyle, personal and family history of disease and risk factors, psychosocial factors, stress, physical activities, history of smoking and alcohol, blood physiological and biochemical parameters.

\subsection{Genetic variation}

SNPs and Haplotype were explored in two regions (1q42.13 near GALNT2 and 19q13.2 near APOE). Among variants, SAS/genetics was applied to choose maximally informative sets of SNPs and elaborate genetic variations by linkage disequilibrium and $D^{\prime}$ of 0.90 .

\subsection{Blood collection and genotyping}


Non-fasting blood samples $(20 \mathrm{ml})$ were collected and centrifuged within $2 \mathrm{~h}$ of admission and frozen quickly after centrifugation. All the blood samples were transferred in nitrogen vapor tanks from the sites to a blood storage site and stored at $-70^{\circ} \mathrm{C}$ in freezers or $-170^{\circ} \mathrm{C}$ in liquid nitrogen. All participants had DNA samples available. The GeneAmp 5700 Sequence Detector (Applied Biosystem) was used to genotype participants' SNPs by an allele-specific real-time polymerase chain reaction.

\subsection{Statistical analysis}

Pearson chi-squared analysis also assessed the Hardy-Weinberg equilibrium by the ALLELE procedure in SAS 9.4 version/Genetics. Univariate and multivariable logistic regression models were applied to evaluate genetic associations with hypertension disease for each genetic variant.

\section{Results}

The general and biochemical characteristics between the hypertension $(N=211)$ and normal populations $(N=434)$ were indicated in Table 1 .

The genotypic frequencies of both SNPs all followed in Hardy-Weinberg equilibrium. The AA, AG, and GG genotype frequencies of GALNT2 rs 4846914 were $22.8 \%, 43.1 \%$, and $34.1 \%$ in hypertension subjects, and $35.3 \%, 44.2 \%$ and $20.5 \%$ in controls $(P<0.05)$, respectively (Table 2$)$. The A and $\mathrm{G}$ allele frequencies of SNP rs 4846914 near GALNT2 were $44.3 \%$ and $55.7 \%$ in hypertension cases and $57.4 \%$ and $42.6 \%$ in controls $(P<0.05)$, respectively. After being adjusted, the occurrence of OR of AG genotype compared with AA genotype was 1.61 (95\% CI: 1.02 to 2.56$)$ and 2.67 (95\% CI: 1.59 to 4.488) for the GG genotype. SNP rs429358, rs7412, and rs405509 genotype frequencies near APOE between hypertension and control subjects were no significant statistical difference (Table 2).

Table 1

Characteristics of participants

\begin{tabular}{|c|c|c|c|c|}
\hline Characteristics & $\begin{array}{l}\text { Hypertension cases, } \\
N=211\end{array}$ & controls, $N=434$ & $\chi^{2 / t}$ & $P$-value \\
\hline Male sex $(\%)$ & $134(63.5)$ & $316(72.8)$ & 5.83 & 0.0158 \\
\hline Mean Age (SD), year & $64.9(10.2)$ & $60.0(10.5)$ & 5.53 & 0.0001 \\
\hline BMI, mean (SD), $\mathrm{kg} / \mathrm{cm}^{2}$ & $25.2(2.8)$ & $24.0(2.9)$ & 5.49 & 0.0001 \\
\hline WHR, mean $(\mathrm{SD}), \mathrm{cm} / \mathrm{cm}$ & $0.90(0.09)$ & $0.86(0.08)$ & 4.70 & 0.0001 \\
\hline Systolic blood pressure $(\mathrm{mmHg})$ & $142.3(17.9)$ & $123.1(12.7)$ & 13.34 & 0.0001 \\
\hline Diastolic blood pressure $(\mathrm{mmHg})$ & $86.1(10.7)$ & $77.5(7.5)$ & 10.06 & 0.0001 \\
\hline Diabetes $(\%)$ & $23(10.9)$ & $5(1.2)$ & 32.49 & 0.0001 \\
\hline Stroke $(\%)$ & $25(11.9)$ & $8(1.8)$ & 29.28 & 0.0001 \\
\hline Smoking, n (\%) & $78(37.0)$ & $192(39.6)$ & 0.42 & 0.5146 \\
\hline Alcohol, n (\%) & $23(10.9)$ & $63(14.5)$ & 1.61 & 0.2050 \\
\hline General stress, n (\%) & & & 8.15 & 0.0170 \\
\hline Permanent & $77(36.5)$ & $114(26.3)$ & & \\
\hline Several periods & $88(41.7)$ & $226(52.1)$ & & \\
\hline Never experienced & $46(21.8)$ & $94(21.7)$ & & \\
\hline ApoA1/ApoB & $1.60(0.45)$ & $1.80(0.59)$ & -4.66 & 0.0001 \\
\hline
\end{tabular}

Note: $N$, number; $p$-value, significance of difference between group means or frequencies determined by $t$-test or chi-square test. 


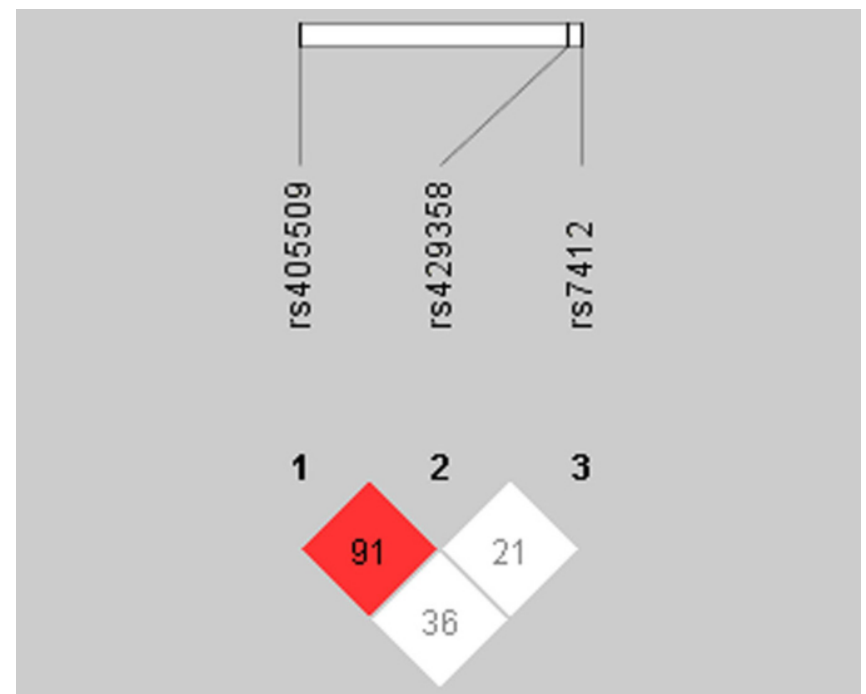

Fig. 1. Pair-wise LD among three SNPs in $19 \mathrm{q} 13.2$ in the control group. The numbers are $D^{\prime} \times 100$

Table 2

Four SNPs genotype and their frequencies

\begin{tabular}{|c|c|c|c|c|c|c|c|c|c|}
\hline SNP & $\begin{array}{l}\text { Case } \\
\text { count }\end{array}$ & $(\%)$ & $\begin{array}{l}\text { Control } \\
\text { count }\end{array}$ & $(\%)$ & $\mathrm{Chr}$ & Pos & Nearby gene & Crude OR(CI) ${ }^{*}$ & $\begin{array}{l}\text { Adjusted } \\
\text { OR(CI) }{ }^{* *}\end{array}$ \\
\hline rs4846914 & & & & & $1 \mathrm{q} 42.13$ & 228362314 & GALNT2 & & \\
\hline AA & 48 & 22.8 & 153 & 35.3 & & & & 1.00 & 1.00 \\
\hline $\mathrm{AG}$ & 91 & 43.1 & 192 & 44.2 & & & & $1.51(1.00-2.27)$ & $1.61(1.02-2.56)$ \\
\hline GG & 72 & 34.1 & 89 & 20.5 & & & & $2.58(1.65-4.04)$ & $2.67(1.59-4.48)$ \\
\hline rs429358 & & & & & $19 \mathrm{q} 13.2$ & 42216263 & APOE & & \\
\hline AA & 177 & 84.3 & 352 & 81.1 & & & & 1.00 & 1.00 \\
\hline AG & 33 & 15.7 & 77 & 17.7 & & & & $0.85(0.55-1.33)$ & $0.82(0.50-1.34)$ \\
\hline GG & 0 & 0 & 5 & 1.2 & & & & $\ldots$ & $\ldots$ \\
\hline rs7412 & & & & & $19 q 13.2$ & 42216401 & APOE & & \\
\hline $\mathrm{AA}$ & 199 & 94.3 & 423 & 97.5 & & & & 1.00 & 1.00 \\
\hline AG & 12 & 5.7 & 11 & 2.5 & & & & $2.32(1.01-5.35)$ & $1.37(0.52-3.58)$ \\
\hline rs405509 & & & & & $19 q 13.2$ & 50100676 & APOE & & \\
\hline $\mathrm{CC}$ & 15 & 7.1 & 30 & 7.0 & & & & 1.00 & 1.00 \\
\hline $\mathrm{AC}$ & 74 & 35.1 & 161 & 37.4 & & & & $0.92(0.47-1.81)$ & $0.88(0.41-1.89)$ \\
\hline AA & 122 & 57.8 & 239 & 55.6 & & & & $1.02(0.53-1.97)$ & $0.84(0.40-1.78)$ \\
\hline
\end{tabular}

Note: Chr: Chromosome; Pos: position; * Crude Odds ratio (OR) of being influenced by a SNP for an expression trait associated with a given risk factor; ** Adjusted for sex, age, BMI, smoking, drink, Diabetes, Stroke history, stress, Apoa1, and Apob.

Figure 1 indicated the results of haplotype analysis for the examined SNPs. Next, we used haploview and SAS software to calculate possible haplotype frequency of 3 loci in 19p13.2. Using the genotypes of 434 controls, we defined the haploblock structure of SNPs within the region of 19p13.2 in the Chinese population. By defining a solid spine of LD as $D^{\prime}>0.90$, we found one haploblock in the 19p13.2 region (Figure 1). Next, we focused on the association of haplotypes within one block. Our results indicate that there is no significant association between haploblock on chromosome 19 p13.2 and hypertension $(P=0.3430)$.

Table 3 indicates the stratification analysis result of rs 4846914 and its association with hypertension. In models adjusted for all the risk factors, OR (rs4846914 GG genotype compared to AA) associated 
with BMI was 1.16 (95\% CI: 0.51 to 2.63$)$ in the group where $\mathrm{BMI}<24$, and 4.15 (95\% CI: 2.08 to 8.27 ) in the group where $\mathrm{BMI} \geqslant 24$ ( $p$ value for heterogeneity, 0.1096).

Table 3

Stratification analysis for association between rs4846914 genotypes and hypertension

\begin{tabular}{|c|c|c|c|c|}
\hline & \multicolumn{4}{|c|}{ rs 4846914 OR $(95 \%$ CI) } \\
\hline & $\mathrm{AA}$ & $\mathrm{AG}$ & GG & $P$-value \\
\hline$\overline{\mathrm{BMI}}$ & & & & 0.1069 \\
\hline$<24$ & 1.00 & $1.29(0.62-2.66)$ & $1.16(0.51-2.63)$ & \\
\hline$\geq 24$ & 1.00 & $1.83(1.01-3.31)$ & $4.15(2.08-8.27)$ & \\
\hline Smoking & & & & 0.5492 \\
\hline No & 1.00 & $1.52(0.85-2.70)$ & $2.55(1.35-4.83)$ & \\
\hline Yes & 1.00 & $2.01(0.88-4.57)$ & $3.43(1.35-8.70)$ & \\
\hline General stress & & & & 0.0132 \\
\hline Several or never & 1.00 & $1.59(0.94-2.70)$ & $2.06(1.07-3.95)$ & \\
\hline Permanent & 1.00 & $2.27(0.77-6.65)$ & $4.99(1.69-14.75)$ & \\
\hline
\end{tabular}

\section{Discussion}

Hypertension disease is one of most serious risk factor for CVD, stroke, and end-stage renal disease. Hypertension is a global problem; thus, prevention of hypertension remains a global public health goal. The potential association between the GALNT2 polymorphisms and plasma or serum lipid levels in humans has been proved by several previous GWAs studies. These researches have confirmed that the minor allele of GALNT2 polymorphisms was related to low HDL-C and high TG blood levels [11-14].

In the current study, we found a significantly higher promoter methylation of GALNT2 in the hypertension group than in the non-hypertension group. The hypertension participants also had higher GG genotype frequency of rs 4846914 then the normal individuals. Statistically significant evidence of the whole sample indicated that the GALNT2 gene promoter hypermethylation increases the risk of hypertension $(\mathrm{OR}=1.51 ; 95 \% \mathrm{CI}, 1.00-2.27 ; P<0.05$ and $\mathrm{OR}=2.58 ; 95 \% \mathrm{CI}, 1.65-4.04 ; P<0.001)$. After other risk facts were adjusted for, similar results were obtained $(\mathrm{OR}=1.61 ; 95 \% \mathrm{CI}, 1.02-2.56$; $P<0.05$ and $\mathrm{OR}=2.67 ; 95 \% \mathrm{CI}, 1.59-4.48 ; P<0.001)$. However, other genetic and environmental factors might affect levels of relationship. The hypertension participants with the GG genotype of rs4846914 had higher ApoB levels and lower ApoA1 levels than the normal participants; the ratio of ApoA1 to ApoB was also lower. A previous candidate gene study can not find any effect of the GALNT2 rs4846914 variant on serum TC and TG levels, and any of these findings were not authenticated by a meta-analysis of the six studies. This may be due to the modest effects on lipid concentrations of these variants and lower power of statistics for testing the relationship [16]. However, levels of LDL-C were related to the genotypes of rs4846914 in the Chinese Han population. Still, several GWAs and candidate gene researches have failed to find a significant relationship between GALNT2 polymorphisms and hypertension.

It is well established that environmental factors such as obesity, physical activity, stress, lifestyle, and dietary patterns are all strongly correlated with hypertension. This study indicated that rs 4846914 was related to age, sex, cigarette smoking, BMI, and general stress in two groups. These data suggest that environmental factors also play an important role in determining hypertension risk in the China Han population. In lower BMI groups, the association between rs4846914 and hypertension was not 
statistically significant. However, in the higher BMI group, the OR was higher, although the heterogeneity analysis was not significant. In the permanently stressed group, the influence of rs4846914 on hypertension was higher than occasionally or never stressed group, $P=0.0132$.

Accumulating evidence reveals that the polymorphism of the APOE gene is genetically associated with many diseases, including hypertension, coronary artery disease, polycystic ovary syndrome, Alzheimer's disease, psoriasis, vascular dementia, gallbladder stone disease, and cerebrovascular disorders. We studied the correlation between the APOE gene and susceptibility to hypertension in China Han population by determining the polymorphism of 3 SNPs, rs429358, rs7412, and rs 405509 . The results demonstrated that there was no significant statistical difference in the genotype and allele distributions of SNPs rs429358 and rs405509 between the hypertension patients and non-hypertension controls. However, the distributions of the genotypes and alleles of the SNP rs 7412 were significantly different between the hypertension patients and normal subjects $(\mathrm{OR}=2.32, P<0.05)$. But when we adjusted for other risk factors, we found no convincing association between the SNP rs7412 and hypertension risk. Some previous studies have suggested that polymorphism at $+2836 \mathrm{G}>\mathrm{A}$ of the APOE gene is strongly related to the susceptibility to hypertension; the A allele of the APOE gene may be a risk factor for hypertension in Chinese Hui individuals while the $G$ allele may serve as a protective factor for hypertension. This genetic variation at the $+2836 \mathrm{G}>\mathrm{A}$ site of the APOE gene was first described by Matsunaga et al. [17] who demonstrated that the G>A mutation changed glutamic acid into a lysine residue. Additionally, polymorphisms of the APOE gene have also been correlated with plasma lipid levels and susceptibility to hypertension in a variety of ethnic groups, although there have been inconsistent results [18].

Some limitations of our research should be taken into consideration. First, a potential disadvantage was that the control participants may not represent the general China Han population, nor were the hypertension subjects representative of all China hypertension patients. Another was that we did not collect enough samples to prove the association between gene SNPs and hypertension. Additionally, our study lacked a strict control matching process and there was selection bias.

\section{Conclusion}

Our present work provides evidence to support the relationship of gene SNPs with the risk profile of hypertension. Our data indicates that SNP rs4846914 in GALNT2 gene is related to an increased risk of hypertension in China population, but the APOE gene is not. ApoA1, BMI, and general stress are also likely to be important factors influencing hypertension.

\section{References}

[1] High blood pressure a growing threat to China's public health Nation, Jul. $7^{\text {th }}, 2013$, http://www.chinadaily.com.cn/cndy/2013-04/08/content16381678. htm.

[2] Nation Center for Cardiovascular Diseases: Report on Cardiovascular Disease in China, Beijing, 2007.

[3] B.M. Egan and Y. Zhao and R.N. Axon, US trends in prevalence, awareness, treatment, and control of hypertension, 1988-2008, Journal of the American Medical Association 303 (2010), 2043-2050.

[4] A.G. Holleboom, M. Vergeer, G.K. Hovingh, J.J. Kastelein and J.A. Kuivenhoven, The value of HDL genetics, Current Opinion in Lipidology 19 (2008), 385-394.

[5] T.M. Teslovich, K. Musunuru, A.V. Smith, A.C. Edmondson, I.M. Stylianou, M. Koseki, et al., Biological, clinical and population relevance of 95 loci for blood lipids, Nature 466 (2010), 707-713.

[6] E.S. Tai, X.L. Sim, T.H. Ong, T.Y. Wong, S.M. Saw, T. Aung, et al., Polymorphisms at newly identified 
lipid-associated loci are associated with blood lipids and cardiovascular disease in an Asian Malay population, Journal of Lipid Research 50 (2009), 514-520.

[7] K. Musunuru, M. Orho-Melander, M.P. Caulfield, S. Li, W.A. Salameh, R.E. Reitz, et al., Ion mobility analysis of lipoprotein subfractions identifies three independent axes of cardiovascular risk, Arteriosclerosis Thrombosis and Vascular Biology 29 (2009), 1975-1980.

[8] A.C. Edmondson, P.S. Braund, I.M. Stylianou, A.V. Khera, C.P. Nelson, M.L. Wolfe, et al., Dense genotyping of candidate gene Loci identifies variants associated with high-density lipoprotein cholesterol, Circulation. Cardiovascular Genetics 4 (2011), 145-155.

[9] S. Kathiresan, O. Melander, C. Guiducci, A. Surti, N.P. Burtt, M.J. Rieder, et al., Six new loci associated with blood lowdensity lipoprotein cholesterol, high-density lipoprotein cholesterol or triglycerides in humans, Nature Genetics $\mathbf{4 0}$ (1998), 189-197.

[10] J. Wang, M.R. Ban, G.Y. Zou, H. Cao, T. Lin, B.A. Kennedy, et al., Polygenic determinants of severe hypertriglyceridemia, Human Molecular Genetics 17 (2008), 2894-2899.

[11] N. Polgár, L. Járomi, V. Csöngei, A. Maász, C. Sipeky, E. Sáfrány, et al., Triglyceride level modifying functional variants of GALTN2 and MLXIPL in patients with ischaemic stroke, European Journal Neurology 17 (2010), 1033-1039.

[12] C.A. Reynolds, M.G. Hong, U.K. Eriksson, K. Blennow, F. Wiklund, B. Johansson, et al., Analysis of lipid pathway genes indicates association of sequence variation near SREBF1/TOM1L2/ATPAF2 with dementia risk, Human Molecular Genetics 19 (2010), 2068-2078.

[13] R. Dawar, A. Gurtoo and R. Singh, Apolipoprotein A1 gene polymorphism (G-75A and C+83T) in patients with myocardial infarction: A pilot study in a north Indian population, American Journal Clinical Pathology 134 (2010), 249-255.

[14] J. Scott, T.J. Knott, D.J. Shaw and J.D. Brook, Localization of genes encoding apolipoproteins CI, CII, and E to the p13 $\rightarrow$ cen region of human chromosome 19, Journal of Human Genetics 71 (1985), 144-146.

[15] R.W. Mahley, Apolipoprotein E: Cholesterol transport protein with expanding role in cell biology, Science 240 (1988), 622-630.

[16] D. Chasman, G. Pare and P. Ridker, Population-based genomewide genetic analysis of common clinical chemistry analytes, Clinical Chemistry 55 (2009), 39-51.

[17] A. Matsunaga, J. Sasaki, K. Moriyama, F. Arakawa, Y. Takada, K. Nishi, et al., Population frequency of apolipoprotein E5 (Glu3 $\rightarrow$ Lys) and E7 (Glu244 $\rightarrow$ Lys, Glu245 $\rightarrow$ Lys) variants in western Japan, Clinical Genetics 48 (1995), 93-99.

[18] C.F. Liu, Q.F. Yang, X.L. Chen and C.Y. Liu, Apolipoprotein a5 gene polymorphism and risk for metabolic syndrome: A meta-analysis, Genetic Testing and Molecular Biomarkers 16 (2012), 1241-1245. 